\title{
Prevalence of Low Back Pain in Pregnant Women and the Associated Risk Factors
}

\section{Gharaibeh $\mathrm{A}^{1 *}$, Al Wadiya $\mathrm{A}^{2}$, Qdhah $\mathrm{E}^{3}$, Khadrawi $\mathbf{M}^{4}$, Abu Slaih $\mathrm{A}^{4}$ and Qaoud $\mathrm{Y}^{5}$}

${ }^{1}$ Orthopaedic Surgeon, Department of Orthopaedics and musculoskeletal trauma, Louis Pasteur University, Slovakia

${ }^{2}$ Orthopaedic Surgeon, Royal Derby Hospital, UK,

${ }^{3}$ Private clinic, Irbid, Jordan,

${ }^{4}$ Ministry of Health, Amman, Jordan

\section{Research Article}

Volume 2 Issue 2

Received Date: April 25, 2018

Published Date: May 09, 2018

4Ministry of Health, Amman, Jordan

${ }^{5}$ Department of Public Health, Jordan University of science and technology, Jordan

*Corresponding author: MUDr. Ahmad Gharaibeh, PhD, MPH, Orthopaedic Surgeon, Department of Orthopaedics and musculoskeletal trauma, Louis Pasteur university Hospital, LF UPJS Kosice, Slovakia. Tel: +421915344317; Email: gharaibeh@seznam.cz

\section{Abstract}

Objective: This study aimed to compare and analyse prevalence of low back pain during pregnancy. We looked into the risk factors, impact on everyday life and estimate the severity of the problem in major maternity and childhood centers in Jordan.

Design: This is a cross-sectional study that was performed on pregnant women attending prenatal care and various maternity and childhood centers. They have been asked to fill a structured and authored questioner about lower back pain.

Results: The study was conducted on 408 pregnant women. These women attended the three largest maternity and childhood centers in Jordan. 310 pregnant women had lower back pain $(76 \%)$ and 98 pregnant women $(24 \%)(\mathrm{P}<0.01)$ had no pain. Higher age, post term date, high BMI and long working hours are statistically significant criteria for the back pain symptoms in pregnant women.

Conclusions: The results show that lower back pain during pregnancy is a common problem in this population. Low back pain causes sleep disturbances and mood swings in pregnant women.

Keywords: Prevalence; low back pain; Pregnancy; Risk factors; Epidemiology 


\section{Journal of Orthopedics \& Bone Disorders}

\section{Introduction}

World Health Organisation (WHO) definition of low back pain (LBP) is symptom of various cases involving the spine. Low back pain or pelvic pain (LBPP) during pregnancy was defined as "recurrent or continuous pain for more than 1 week from the lumbar spine or pelvis" during actual pregnancy (5). Despite modern diagnostic methods, they are nonspecific in the diagnosis of many cases. Classification of LBP according to WHO in 2013: Subacute 6-12 weeks, Acute less than 6 weeks, Chronic longer than 7- 12 weeks [1-3]. In general, lumbar pain during pregnancy is similar to low back pain experienced by non-pregnant women. This type of pain typically increases with prolonged postures (such as sitting, standing, or repetitive lifting). Tenderness may also be present in the muscles along the spine during pregnancy $[1,3]$.

The literatures shows that a study done in 2007 in the USA on 599 pregnant women all of them in the second trimester, that $67 \%$ of pregnant women had low back pain, $75 \%$ of them had painkillers, and $85 \%$ of those did not reduce their pain [4]. And In a study conducted in Sweden in 2005 on 891 pregnant women attending women's clinics and maternity, shows that $72 \%$ suffers from lower back pain .Factors associated with the low back pain among pregnant women, are history of hypermobility, High Body mass index (BMI) history of menopause, and previously suffering from low back pain during other activities [5]. Another study conducted in Brazil in 2005 on 203 pregnant women arriving to the prenatal care at Basic Health Units of the city of Paulínia showed that back pain was in $79.8 \%$ of the attenders. $80.8 \%$ suffer from low back pain in the lumbar region and $49.1 \%$ suffer from pain in the sacroiliac joints and it was found that younger pregnant women had more frequent pain [6]. In a study conducted in Australia in 1996 on pregnant women attending rural general practice for antenatal care showed that 20 pregnant women suffers from low back pain and it is clear that the problem of lower back pain is the result of dysfunction of iliolumbar ligament. Dysfunction was identified in three while the remaining had sacroiliac joint dysfunction which is the source of pain in the pregnant women. The study found that 15 patients had no pain after 3 visits and the rest had more than 50\% improvement in their pain after treatment with movement and home exercises [7]. Another study conducted in occupied Palestine in 1994 on 449 pregnant showed that $54.8 \%$ of them had lower back pain. The most contributing factor associated with the increased lower back pain during pregnancy is low socioeconomic class [8]. In the 1993 study in Taiwan, 200 normal pregnancies 24 - 48 hours postpartum showed that $54.5 \%$ had symptoms of low back pain during pregnancy [9]. In a Swedish study in 1991, 429 pregnant women with a history of low back pain and 375 pregnant women who had no history of low back pain were seen on regular bases from the beginning of the 12th week until the childbirth. Lower back pain was found to be twice as high in women with a history of low back pain. Younger Pregnant women had higher incident of low back pain in the $1^{\text {st }}$ trimester but improved by the end of pregnancy [10]. In another study, conducted in Sweden in 1991 on 855 pregnant women from the beginning of the twelfth week until childbirth showed that $49 \%$ of lower back pain patients had it in the ninth month. $27 \%$ of the patients had it in the sixth month and in the third month $22 \%$ of the patients were found with lower back pain. More likely to have low back pain are younger pregnant women, those multiple pregnancies and those with several physical and psychological work [11]. In a cohort study conducted in Sweden in 1996 on 200 pregnant women showed that the incidence of low back pain was $76 \%$ reported low back pain, the rate to $48 \%$ until the twenty fourth weeks and after birth incidence of pain decreased to $9.4 \%$ [12]. In the study conducted in Canada on 400 pregnant show that the incidence of pain lower back was $42.5 \%$ during pregnancies and $44.7 \%$ during childbirth [13], and in a study conducted in Iran on 161 pregnant women out of 325 showed that the incidence of low back pain was $49.5 \%$ [14], and in a study Conducted in Sweden on 313 pregnant women showed that the incidence of low back pain related to pelvic girdle was $54 \%, 17 \%$ is lumbar pain and $29 \%$ of pain was a combination of both [15]. In a review study in 2017 confirmed that a potential for increased risk of neurological complications in pregnant women with pre-existing neurological deficits before pregnancy [16], and finally Sklempe confirmed that physical therapy reduces the low back pain in pregnancy $[17,18]$.

The importance of the subject: Back pain is one of the most common health problems in the world, where patients with lower back pain are more likely to visit orthopaedic clinics and physiotherapy. Where $80 \%$ of adults suffer from low back pain once or more during their lives (four out of every five people), is one of the most common causes of absence from work as it is the second reason after colds and flu [1-3]. Knowing the prevalence of low back pain and related risk factors helps to identify the size of the problem and identify appropriate recommendations to contribute to reducing the direct and indirect causative factors to reduce disability and alleviate the pain caused by complications of low back pain in pregnant women and raise the standard of living. Also we have to recognize that the inability to give pregnant women strong analgesic and 


\section{Journal of Orthopedics \& Bone Disorders}

muscle relaxants, due to side effect on the fetus. The results of this study are expected to contribute to providing an information base that helps planners and decision makers in developing appropriate strategies to deal with this Health as a whole.

\section{Methodology}

Study Design: A cross-sectional study to answer a research questioner about the prevalence of low back pain and its risk factors among pregnant women. The results and necessary comparisons analyzed within the context of the study by selecting pregnant women who visit Maternal and Child Centers in comprehensive centers in Northern Jordan.

Place of study: There are 385 maternity and childhood centers in Jordan, of which 152 are in Northern Jordan and 40 are in Irbid Health Directorate. The three largest maternity and childhood centers in the Irbid Health Directorate were selected by cluster sampling in terms of number of visits in Irbid to conduct research. The health centers of Irbid in 2006 of the new reviews 5605 and the frequent reviews 18220. The comprehensive centers include clinics for family medicine, dental clinics; clinics of dermatology, gynecology and obstetrics clinics and emergency rooms, centers contain a laboratory and diagnostic radiation and pharmacy and one ambulance for each center in addition to centers of motherhood and childhood.

Comprehensive health centers:

$>$ Ibn Sina Comprehensive Health Center 334 new reviews and 1208 frequent reviews for treatment renewal and surveillance.

> Kafr Yuba Comprehensive Health Center 242 new reviews and 979 recurrent reviews for treatment renewal and monitoring.

> Sarih Comprehensive Health Center 368 new reviews and 1336 recurrent reviews for treatment renewal and monitoring.

The three centers of motherhood and childhood were chosen because they are the three largest centers in the north of Jordan. They are accredited to train the medical staff of the Jordanian Medical Council, the Jordanian Ministry of Health, the Health Academy, and the presence of sufficient space to receive a large number of participants. The two largest hospitals in the north are Princess Badea Educational Hospital for Obstetrics and Gynecology, a state-run referral hospital and a hospital of the Faculty of Medicine at the University of Science and Technology, King's Founder Hospital. The centers are close to and supported by the Faculty of Medicine As well as their proximity to the research team.

It is a cross sectional study, that was conducted on pregnant women who participated in the examination in the prenatal care. A questionnaire with 49 questions was structured and completed by the authors.

Study population and sample: All pregnant women are referred to clinics for maternity and childhood centers

Aim of our study find out the prevalence of low back pain in pregnant women.

To show that in spite of the high incidence of low back pain in pregnant women, it is a neglected problem.

The aim of our work was to investigate incidence of low back pain during pregnancy, its risk factors, impact on life style, and the seriousness of the problem

Place of research: The three largest centres "for mother and child care" in the north of Jordan, which took place in 2008 with 3523 examinations, we picked randomly 408 patients with low back pain within 8 weeks.

\section{Study Questions}

-What is the prevalence of low back pain among pregnant women in Northern Jordan?

- What are the factors associated with lower back pain among pregnant women in Northern Jordan?

Variables: Independent factor: Pregnancy trimester (First, second, third), age, level of education, income, health insurance, family history of illness, smoking, alcohol, overweight, obesity and chronic diseases such as diabetes and stress (Figure 1).

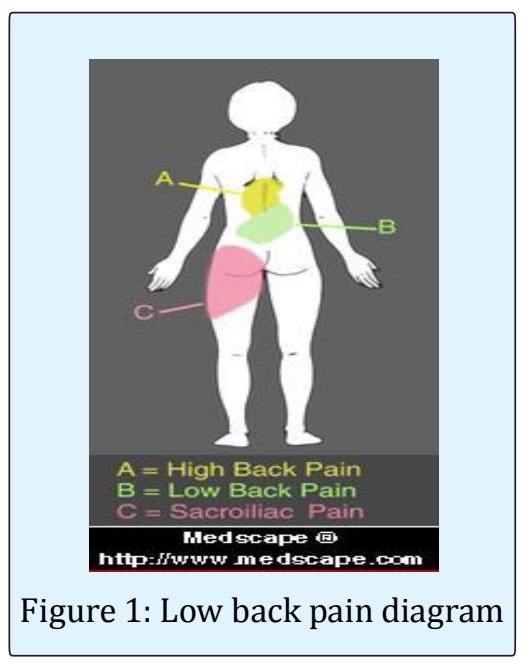

Gharaibeh A, et al. Prevalence of Low Back Pain in Pregnant Women and the Associated Risk Factors. J Ortho Bone Disord 2018, 2(1): 000157.
Copyright(C) Gharaibeh A, et al. 


\section{Journal of Orthopedics \& Bone Disorders}

Ethical and official considerations: Approvals was taken from the Director of Health and from the directors of the health centres and the consent of the participants to the study was signed. All information is confidential and published collectively and any pregnant women can withdraw from the study whenever she wishes and we follow the ethics of scientific research.

Limitation of study: Bad weather in the first week prevented pregnant women from attending their appointments, but on the other hand the attendance in the following two weeks is much larger than expected in the centres of Ibn Sina and Kafr Yuba.

The expected number of pregnant women was much lower than expected and announced in the annual report of the Ministry of Health 5-6 reviews daily and $66 \%$ of the expected number. 6 pregnant women refused to join the study in Al Sareeh centre and 11 questionnaires were almost empty in the Al Sareeh and Ibn Sina centres.

\section{Results}

The work presented prospected randomized study to evaluate the Incidence, reasons and complication of low back pain. We prospectively studied 425 pregnant women. , 310 respondents had pain in the lower spine (76\%). 98 respondents did not have pain $(24 \%)(\mathrm{p}<0.01)$. Randomly we selected pregnant women during the time of our study. All pregnant women were clinically examined before administration and the questioner was filled by researchers and midwifes. 6 pregnant women refused to join the study and 11 questionnaires were almost empty (Graph 1).

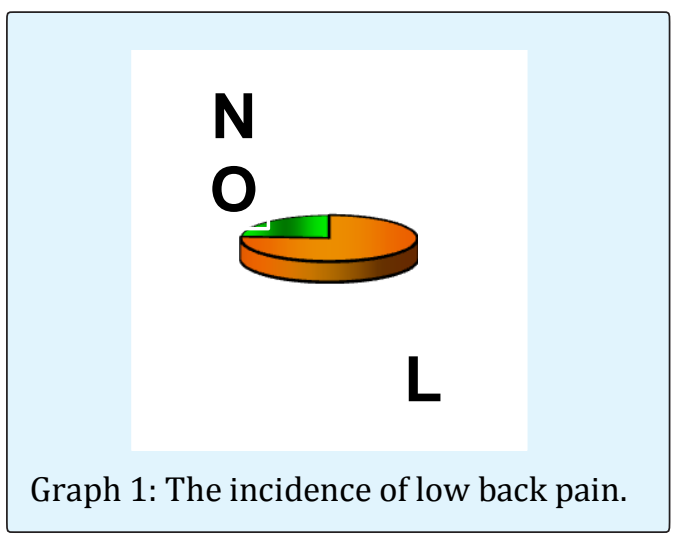

All pregnant women prior to participation in the study were informed about the investigations and questioner and signed informed consent.

Two independent statistic analysts using MS Excel carried out statistical analysis.

425 patients answered the questionnaire (Table 1). The mean age was 26.2 years; high age of pregnant women compared to pain was statistically significant. The median gestational age was 20 weeks. Average weight was 66.4 kilo grams standard deviation $9.123(54-90 \mathrm{~kg})$

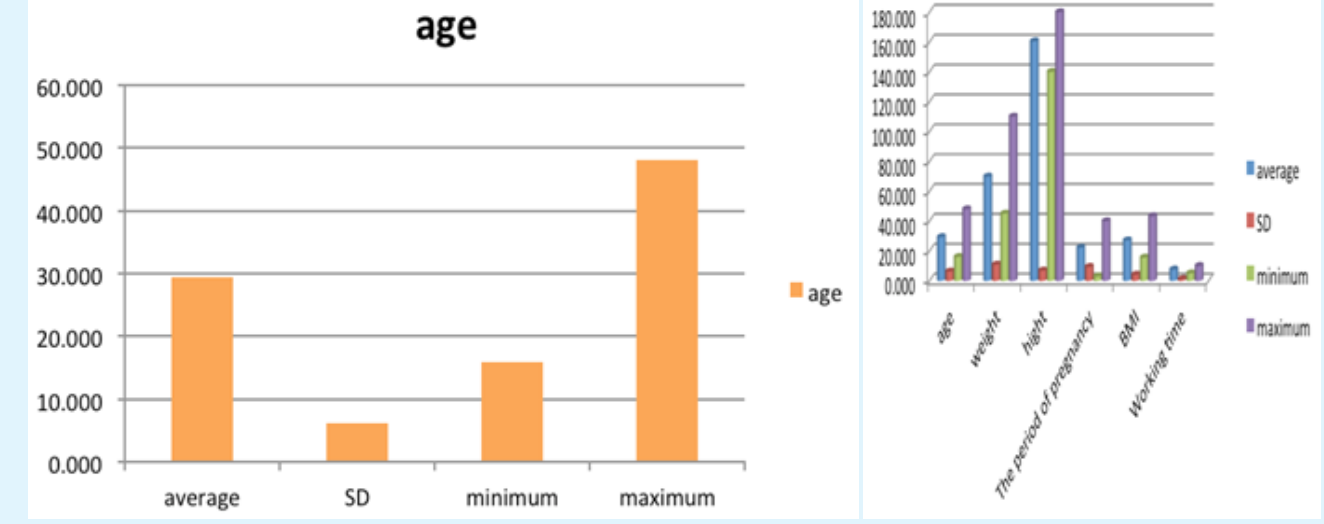

Table 1: The age indicates with low back pain.

Body mass index compared to pain was statistically significant $\mathrm{p}<0.05$.
Working time average for pregnant women was 7.568 hours standard deviation 1.217 (5-10 hours), which is not statically significant. 


\section{Journal of Orthopedics \& Bone Disorders}

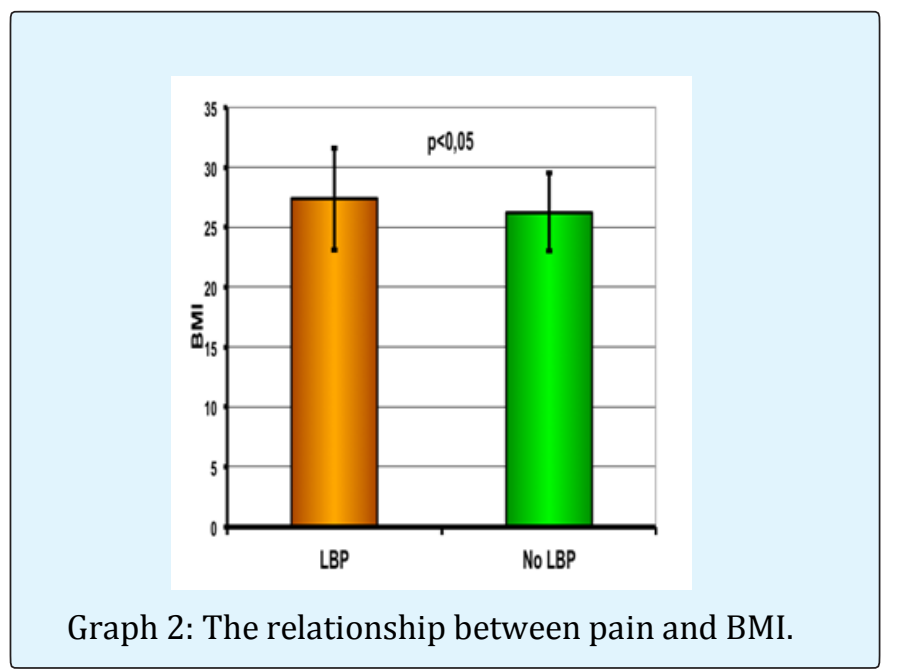

Pregnant women reported low back pain with median pregnancy period 22,8 weeks standard deviation 9.366 (3-40 weeks)and of these $43.9 \%$ reported that low back pain began in the end of second trimester.

History of low back pain in previous pregnancies or without is not statically significant (Graph 2).

Body mass index compared to pain was statistically significant $\mathrm{p}<0.05$

Relation between pain and few psychological parameters: Agitation, spontaneous sleep, sweating, compunction, and early morning wake (Graf 3,4). Were statistically significant, while anxiety and tension in pregnant women were insignificant.

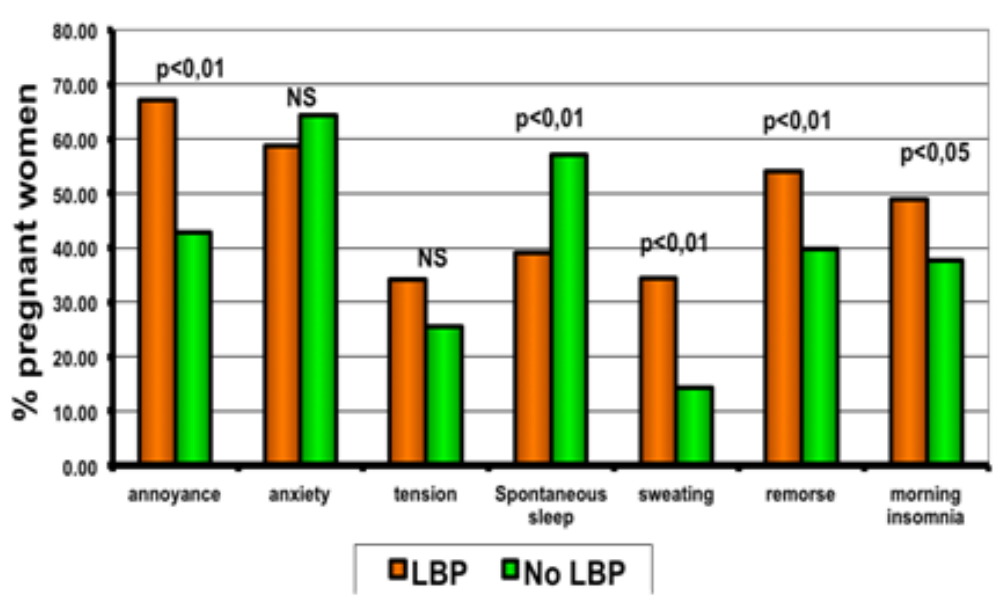

Graf 3: The relationship between Low back pain and few psychological parameters.

Relation between nightmares and LBP was significant in occasionally nightmares $\mathrm{p}<0.01$

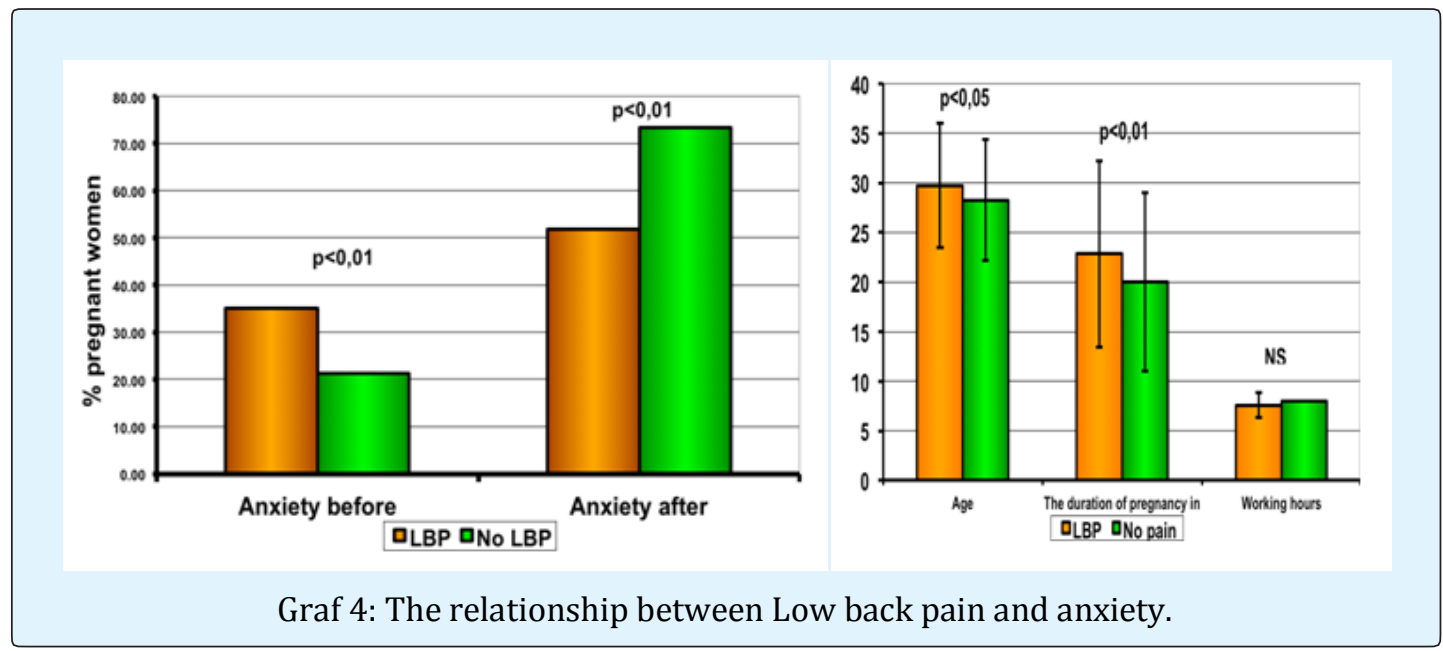




\section{Journal of Orthopedics \& Bone Disorders}

The relation between low back pain and age of pregnant women was significant $\mathrm{p}<0,05$

The relation between low back pain and duration of pregnancy was significant $p<0,01$

The relation between low back pain and working hours was insignificant.

\section{Discussion}

Several studies have shown that there are linear relationship between pain and pregnancy [1-3]. The human pregnancy period involves physical changes. Throughout pregnancy, the woman undergoes physiological changes caused by anatomical and functional needs. Physiological changes affect the musculoskeletal system and usually generate pain, including lower back pain $[1,5]$. In the study which was done 2007 in USA on 599 pregnant women all of them in the second trimester showed that $67 \%$ of pregnant women had low back pain, but in our study it was $76 \%$ as in Sweden $[4,10]$. In the study conducted in Sweden in 2005 on 891 pregnant review women's clinics and maternity it shows that $72 \%$ suffer from lower back pain and factors associated with the pain of the lower back among pregnant women who have a history of increasing traffic and multiple loads and measuring mass index Body mass index (BMI) is high and in our study Body mass index compared to pain was statistically significant [5], as well as in the study conducted in Brazil in 2005 on 203 pregnant women from the center of care for pregnant women showed that $80.8 \%$ suffer from low back pain in the sacral region and in the sacral urethral joints suffer $47.7 \%$ and found that the problem of lower back pain more frequent in Younger pregnant women, but in our study high age of pregnant women compared to pain was statistically significant [6]. In the study, conducted in Sweden in 1991 on 855 pregnant women from the beginning of the twelfth week until birth, where the incidence of pain lower back in the ninth month, $49 \%$, and in the sixth month, $27 \%$ and in the third month, $22 \%$, also in our study the relation between low back pain and duration of pregnancy was significant [11].

This prevalence is considered high, as this is a group of low-risk pregnant women, that is, patients without significant pathological conditions that often worsen back pain, such as obesity, advanced age, and some psychic parameters

\section{Conclusion}

From these results we find out that Low Back Pain is common and serious problem during pregnancy, and causes sleep disturbances. Low back pain cause mood swings with pregnant women. Back pain is one of the most common health problems in the world. Where $80 \%$ of adults suffer from low back pain once or more during their lives (four out of every five people), is one of the most common cause of sick leave as it is the second reason after colds and flu. The results of this study are expected to contribute to providing an information base that helps planners and decision makers in developing appropriate strategies to deal with this Health as a whole. Other studies are recommended for prevention and treatment.

\section{Acknowledgment}

We thank all the staff of medical centres especially midwifes, Irbid, Jordan for their kind support and intensive work and many thanks for our support team Naddaf R., Ababneh M., Raad A., Lataifeh S., Gharaibeh Hanan, Gharaibeh Heba, Sekhnini S, Tawalbeh G., Gharaibeh M.

\section{Interest Conflicts}

The authors declare no conflicts of interest.

\section{References}

1. Solomon L, Warwick D, Nayagam S (2010) Apleys system orthopaedics and fractures. 9th (Edn.), CRC Press Pp: 992.

2. Miller M, Stephen Thompson, Jennifer Hart (2012) Review of Orthopaedics. 6 $6^{\text {th }}$ (Edn.), Philadelphia: Saunders Pp: 896.

3. Dee R (2007) Principles of orthopaedic and fractures. 1522 s. ISBN 0-07-016350-1.

4. Skaggs CD, Prather H, Gross G, George JW, Thompson PA, et al. (2007) Back and pelvic pain in an underserved United States pregnant population: a preliminary descriptive survey. J Manipulative Physiol Ther 30(2): 130-134.

5. Mogren IM, Pohjanen AI (2005) Low back pain and pelvic pain during pregnancy: prevalence and risk factors. Spine 30(8): 983-991

6. Martins RF, Silva JL (2005) Back pain is a major problem for many pregnant women. Rev Assoc Med Bras 51(3): 144-147.

7. McIntyre IN, Broadhurst NA (1996) Effective treatment of low back pain in pregnancy. Aust FAM Physician 25(2): S65-S67. 


\section{Journal of Orthopedics \& Bone Disorders}

8. Orvieto R, Achiron A, Ben-Rafael Z, Gelernter I, Achiron R (1994) Low-back pain of pregnancy. Acta Obstet Gynecol Scand 73(3): 209-214.

9. Fung BK, Kwong CM, Ho ES (1993) Low back pain of women during pregnancy in the mountainous district of central Taiwan. Zhonghua Yi Xue Za Zhi (Taipei) 51(2): 103-106.

10. Ostgaard HC, Andersson GB, (1991) Previous back pain and risk of developing back pain in a future pregnancy. Spine 16(4): 432-436.

11. Ostgaard HC Andersson GB, Karlsson K (1991) Prevalence of back pain in pregnancy. Spine 16(5): 549-552.

12. Kristiansson P, Svärdsudd K, von Schoultz B (1996) Back pain during pregnancy: a prospective study. Spine 21(6): 702-709.

13. Diakow PR Gadsby TA, Gadsby JB, Gleddie JG, Leprich DJ, et al. (1991) Back pain during pregnancy and labor. J Manipulative Physiol Ther 14(2): 116-118.

14. Mousavi SJ, Parnianpour M, Vleeming A (2007) Pregnancy related pelvic girdle pain and low back pain in an Iranian population. Spine 32(3): E100E104.

15. Gutke A, Ostgaard HC, Oberg B (2006) Pelvic girdle pain and lumbar pain in pregnancy: a cohort study of the consequences in terms of health and functioning. Spine 31(5): E149-E155.

16. Sehmbi H, D'Souza R, Bhatia A (2017) Low Back Pain in Pregnancy: Investigations, Management, and Role of Neuraxial Analgesia and Anaesthesia: A Systematic Review. Gynecol Obstet Invest 82(5): 417436.

17. Sklempe Kokic I, Ivanisevic M, Uremovic M, Kokic T, Pisot R, Simunic B (2017) Effect of therapeutic exercises on pregnancy-related low back pain and pelvic girdle pain: Secondary analysis of a randomized controlled trial. J Rehabil Med 49(3): 251-257.

18. World Health Organization, (1995) Third WHO informal consultation of the comprehensive approach to low back pain treatment. 